\title{
Ein Unterschied, der keinen Unterschied macht? \\ Das Signal der Corona Warn-App und die Verwaltung der Pandemie
}

Erschienen in: Sozialfiguren der Corona-Pandemie

Von: Kevin Hall, Klaus Scheuermann, Sven Opitz

Während der ersten Welle der COVID-19-Pandemie wurden große Hoffnungen in das Handlungsvermögen eines technologischen Akteurs gesetzt: die Fähigkeit einer digitalen Smartphone-Anwendung, Kontakte mit Infizierten automatisch nachzuverfolgen und zu melden. Von der Entwicklung dieser Fähigkeit erhoffte man sich ab der zweiten Märzhälfte 2020 die Beantwortung der Frage, wie die Maßnahmen der Bewegungseinschränkung gelockert werden könnten, ohne das Ansteckungsgeschehen erneut anzufachen und die mühsam errungenen Erfolge der Eindämmung zu verspielen. Erschwert wurde die Situation durch ein Sichtbarkeitsproblem: Eine in Science veröffentlichte Studie hatte dargelegt, dass knapp die Hälfte aller SARS-CoV-2Übertragungen präsymptomatisch erfolgt. ${ }^{1}$ Damit erwies sich das „Warnsignal der Symptome“ (ebd.) für die Identifikation als unzureichend, woraus die Notwendigkeit seiner Unterstützung durch digitale Signaltechnologien abgeleitet wurde. Zugleich gewann ein Zeitproblem an Brisanz, denn die Gesundheitsämter beginnen erst mit der Kontaktnachverfolgung, nachdem auf das Auftreten von Symptomen hin ein Test vorgenommen und der positive Befund aus dem Labor empfangen wurde. Aber lässt sich jene Zeitspanne nicht abkürzen, die dann für die Ermittlung von Kontakten im Rahmen von Einzelgesprächen per Telefon benötigt wird? Konstatiert wurde also der Bedarf für einen informationstechnischen Beschleuniger. 


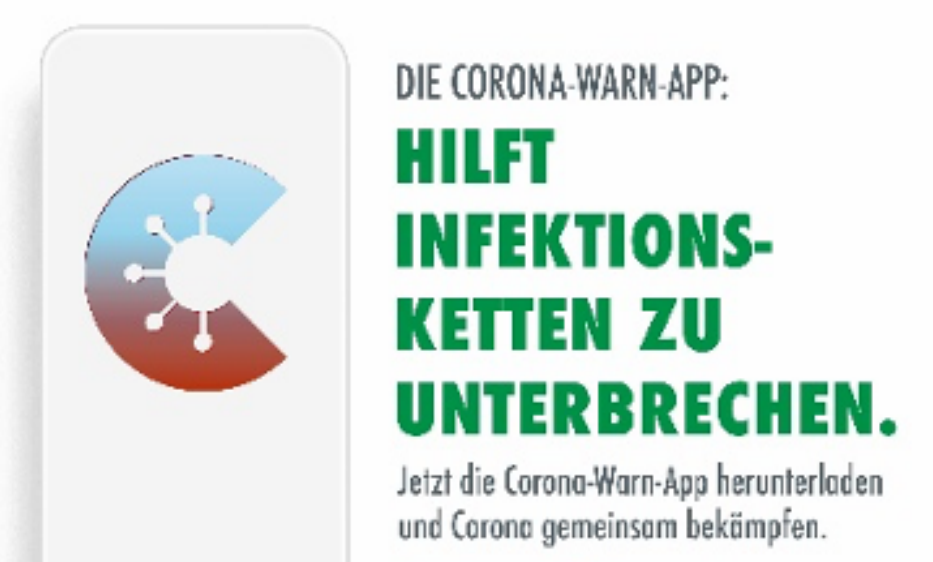

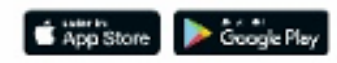

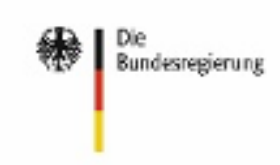

Abb. 1: Bildwortmarke der Bundesregierung ${ }^{2}$

Das, was wir heute als Corona-Warn-App kennen, begann als Entwurf einer Lösung für diesen historisch situierten Problemkomplex. Das klassische Repertoire menschlicher Sozialfiguren, die im Mittelpunkt der bisherigen Beiträge standen, wurde entsprechend erweitert: Die App entstand als Akteursfiktion, an welche Erwartungen in Bezug auf die Unterbrechung von Infektionsketten adressiert wurden, deren technisches Handlungsvermögen aber erst noch zu realisieren war. Das digitale Imaginäre der Pandemiebekämpfung speiste sich zu diesem Zeitpunkt vor allem durch Verweise auf den Vorbildcharakter von technologisch unterstützten Verwaltungspraktiken in ostasiatischen Staaten. Regelmäßig wurde auf Südkorea rekurriert, wo eine App zur Überwachung der Einhaltung von Quarantäneverordnungen im Einsatz war, und auf Singapur, wo am 20. März die Bluetooth-App „TraceTogether" startete. ${ }^{3}$ In Deutschland mehrten sich Stimmen, welche die Einführungen ähnlicher Technologien befürworteten: "So eine digitale Anwendung wäre sinnvoll, um das Virus zielgerichtet einzudämmen", sagte etwa Digitalstaatsministerin Dorothee Bär. ${ }^{4}$ Der Präsident des IT-Verbands Bitkom, Achim Berg, erklärte, eine App werde „dringend gebraucht, um (...) den Restart unseres wirtschaftlichen und gesellschaftlichen Lebens erfolgreich und sicher zu bewältigen“ . ${ }^{5}$ Und RKI-Präsidenten Lothar Wieler sah in Bluetooth-Apps jenes „digitale Tool“, das „den Gesundheitsämtern später natürlich ein viel, viel schnelleres Erkennen von möglichen Kontaktpersonen" ermöglicht. ${ }^{6}$ Von Entwicklern wurden Tracing-Apps dabei gelegentlich als „eine Art elektronische Impfung“ vorgestellt. ${ }^{7}$

In einer Situation, in welcher der pharmaceutical fix eines Impfstoffs noch in weiter Ferne lag, sollte die App somit einen digital fix leisten. Sie sollte die longue durée der Krise nicht aufheben, sondern qualitativ transformieren. Kurz: Man erwartete von ihr, in der 
Pandemie einen Unterschied zu machen, der einen Unterschied macht.

\section{Ein Unterschied, der keinen Unterschied macht?}

In den insgesamt 34 Interviews, die wir in unserem Projekt „Die Verwaltung der Kontaktverfolgung unter COVID-19“ im Herbst 2020 mit 39 Bediensteten aus 17 deutschen Gesundheitsämtern geführt haben, wird die Corona-Warn-App von den Befragten nur äußerst selten ins Gespräch gebracht. Auf direkte Nachfrage erklärt ein Containment Scout ${ }^{8}$ :

„Also, Vorbehalte hier bei uns waren vor allem: ,Was bringt uns die App?' Und da muss man halt sagen: Für unsere eigentliche Kernarbeit ist die App nichts, was wir nutzen können. Sondern ein weiterer Baustein, der eigentlich losgelöst von uns funktioniert.“

Dass das nicht unbedingt eine Kritik an der App beinhaltet, unterstreicht eine Amtsärztin, die sich tendenziell jedoch ähnlich äußert:

„Ich finde es gut, wie es ist. Die App ist uns eben keine Hilfe, aber das ist auch in Ordnung so. Wir machen unsere Arbeit auch so."

Und die Stabsleitung für das Personal im gleichen Amt erläutert:

„Wir können nicht konkret werden, dafür ist es [das Signal der App] zu unkonkret für uns. [... Die App] stört uns jetzt nicht.“

Diese Aussagen sind in Bezug auf eine Informationstechnologie erstaunlich. Folgt man Gregory Batesons berühmter Bestimmung, dann prozessiert jede Informationstechnologie ein Differenz-Intervall. Batesons Beispiel ist der gänzlich analoge Blindenstock, der die Navigation im Raum informiert: Die Wahrnehmung eines Hindernisses wird übersetzt in die Veränderung der Körperbewegung - „a difference that makes a difference". ${ }^{9}$ Demgegenüber scheint das Signal der App auf dem Gesundheitsamt gerade keinen Unterschied zu machen. In der hochgradig volatilen Krisensituation ist die lokale Gesundheitsverwaltung gegenüber der App weitgehend indifferent. Es kommt ohnehin nicht oft vor, dass Bürger*innen den gängigen Meldeweg umkehren und animiert durch ihre App auf eigene Initiative ein Amt kontaktieren, welches folgenreiche Quarantäne-Entscheidungen treffen könnte. In den Fällen, in denen das doch geschieht, versuchen die Ämter zwar zuweilen die Risikowarnung über den Anrufer aufzunehmen und dem Signal nachzuspüren, um möglicherweise doch noch einen Informationswert zu extrahieren:

„Wir fragen: ,War denn irgendwas? Sind Sie irgendwo gewesen?' Wir kennen ja die Fälle. Wir wissen genau, wo Ausbrüche sind.“ (Stabsleitung Personal)

Aber in der Regel führt eine solche Nachforschung nicht zu Personen, aus denen sich belastbare COVID-19-Kontakte generieren ließen, sondern nur zurück zum „,besorgten Bürger" in der Telefonleitung. Auch wenn der Alarm eine*n Nutzer*in zu einem Anruf beim Gesundheitsamt mobilisiert hat, findet er in den Routinen der Gesundheitsverwaltung in der Regel keinen weiterführenden Anschluss. 
Diese Anschlussproblematik lässt sich mithilfe der Verwaltungssoziologie Niklas Luhmanns noch präziser fassen. Die Stärke von Verwaltungen liegt in ihrem Vermögen, auch Krisen routiniert abzuarbeiten. Routinen ergeben sich aus der Implementierung von Entscheidungsprogrammen, die Entscheidungsprämissen zu Bedingungen zusammenfassen, „bei deren Erfüllung eine Entscheidung als richtig gilt“. ${ }^{10}$ Ein „Signal“ wird dazu mit einer Entscheidungsfolge verkoppelt: wenn $\mathrm{x}$, dann $\mathrm{y} .{ }^{11}$ Das Entscheidungsprogramm ist eine konditionale Struktur, die Relevanzgesichtspunkte vorsortiert und für "Grenzen der Variation“ sorgt. ${ }^{12}$ In der COVID-19-Pandmie ist die Verwaltungspraxis durch zwei derartige Verknüpfungen programmiert, die jeweils die häusliche Absonderung zur Folge haben: (1) wenn Infektion, dann Isolation des Indexfalls; und (2) wenn Kontakt der Kategorie 1 mit einem Indexfall, dann Quarantäne der Kontaktperson. In Bezug auf diese konditionalen Strukturen ist das Signal der App kein „programmauslösendes Zeichen“. ${ }^{13}$ Erstens ist es nicht belastbar genug, um die Entscheidungsfolge der Quarantäne zu begründen. Schließlich registriert die App Kontakte zwischen Mobiltelefonen, nicht zwischen Menschen. ${ }^{14}$ Zweitens sind die Entscheidungsfolgen auch deshalb begrenzt, weil man über die App nicht erfährt, wann, wo oder gar mit wem der Kontakt stattgefunden hat. Die App verweist mit ihrem Auswertungsalgorithmus in äußerst generischer Weise auf Kontaktsituationen. Die riskante Kontaktsituation wird signalisiert, gewinnt aber in zeitlicher, räumlicher und sozialer Hinsicht nur äußerst vage Konturen. Diese hochgradig unbestimmte Kommunikation ist es, die das amtliche Konditionalprogramm ins Leere laufen lässt. ${ }^{15}$ Die einzige Chance der Kontaktverfolgung besteht darin, wie ein leitender Mitarbeiter im Interview bestätigte, „bei null anzufangen“.

\section{Selbst(verwaltungs)technologie}

Versteht man Handeln als Fähigkeit, ,jemanden dazu zu bringen, etwas zu tun“, dann ist die Handlungsmacht der Corona-Warn-App auf den Gesundheitsämtern zwar nicht abwesend, aber doch begrenzt. ${ }^{16}$ Ihr Vermögen, riskante Assoziationen zu verzeichnen, übersetzt sich nicht in die eindeutige Konditionierung eines Entscheidungsakts, sondern initiiert bestenfalls allgemeine Nachfragen sowie Weiterverweise an klinische Testzentren oder hausärztliche Praxen. Diese Abwesenheit eines hinreichend bestimmten Anschlusswertes wird Ende 2020 angesichts hoher Infektionszahlen in der Öffentlichkeit zunehmend als Mangel wahrgenommen. Es wird ein Umbau der App gefordert, durch den die Ämter spezifischere Informationen in ihrer Arbeit der Kontaktpersonennachverfolgung erhalten. Auch wenn sich die Schuldzuweisungen im öffentlichen Diskurs auf Softwareunternehmen, ministerielle Entscheidungsträger und rechtliche Regularien erstrecken, geht es letztlich um das Erwartungsmanagement gegenüber einem technologischen Akteur: Die App soll so verändert werden, dass sie bestimmten politischen Erwartungen hinsichtlich der administrativ-technologischen Durchdringung der „infektiösen Sozialität“ im Kontext der Pandemie genügt. ${ }^{17}$

So wird jedoch eine Kontroverse neu eröffnet, die in der Ausgestaltung der Datenarchitektur der App im Frühsommer 2020 bereits beigelegt worden war. Tatsächlich ist der höchst unbestimmte Anschlusswert des Risikosignals auf den Gesundheitsämtern alles andere als ein Planungsfehler oder gar ein Missgeschick. Er 
beruht vielmehr auf der bewussten Entscheidung für eine Datenarchitektur, die auf Prinzipien der Datensparsamkeit, der Anonymität und der dezentralen Speicherung basiert. ${ }^{18}$ Diese Entscheidung war durch hohe Standards im Bereich des Datenschutzes motiviert. Weit davon entfernt, bloß ein hehres, aber in der Pandemiebekämpfung hinderliches Ideal zu verkörpern, hat sich der Datenschutz dabei als funktional erwiesen. Es darf zumindest bezweifelt werden, ob eine weniger datensparsame App bis dato rund 25 Millionen Mal installiert worden wäre. Wer für die Rezentralisierung der Datenarchitektur oder für Datenzugriffe durch die Gesundheitsämter plädiert, um die Anschlussfähigkeit des digitalen Signals zu erhöhen, handelt also unter Umständen riskant. Er riskiert nämlich, dass die Risikotechnologie der App selbst als riskant in Bezug auf die Datensicherheit wahrgenommen und in der Folge von den Benutzer*innen nicht auf das Endgerät geladen, nicht upgedatet, ausgeschaltet oder gar deinstalliert wird. ${ }^{19}$

Anstatt nach dem ultimativen digital fix der Kontaktpersonennachverfolgung zu streben, ist möglicherweise eine Kalibrierung der an den technologischen Akteur gerichteten Erwartungen angezeigt. Offenbar ist die aktuelle App ein Werkzeug, das Dritte nach einem positiven Test vor einer potenziellen Ansteckung warnt und Laborergebnisse an Betroffene übermittelt. Sie etabliert jedoch keine regulären Datenflüsse zwischen Ämtern und Bürger*innen. Man könnte die App damit als eine genuin liberale Technologie begreifen, die das Individuum gerade nicht durch den Staat führt, sondern es in die Verantwortung setzt, das eigene Verhalten an den signalisierten Risikostatus anzupassen. Sie ruft den Einzelnen in bestimmter Weise als „Moralsubjekt" an. ${ }^{20}$ Sie verklammert die Sorge um sich mit der Sorge um andere: Sich selbst vermittelt über das Warnsignal als von Krankheit bedroht wahrzunehmen, geht im Idealfall einher mit der Übernahme von Verhaltensmaximen, welche die Gefährdung anderer minimiert. Die von der Corona-Warn-App initiierte Sorge um sich eröffnet auf diese Weise die Chance zur „Intensivierung der gesellschaftlichen Beziehungen“. ${ }^{21}$

So gesehen erweist sich die Stärke der App in ihrem ganz alltäglichen Anschlusswert. Sie leitet dazu an, sich gegenüber anderen in Vorsicht zu üben. Sie könnte zum Vehikel einer gesellschaftlichen Umsicht werden, für die die Leitung durch das Amt nicht nötig ist. Würde sich diese liberale Auffassung allgemein durchsetzen, dann wären die Ämter ganz offiziell davon entlastet, ihre Routinen von der App informieren zu lassen. Ohne in Rechtfertigungszwänge zu gelangen, könnten sie gegenüber dem digitalen Warnsignal eine eher indifferente Haltung einnehmen und gewännen dadurch Kapazitäten, ihren eigentlichen Kerntätigkeiten sowie anderen Aufgaben nachzugehen. 


\section{c \\ DIE COROHA-WARH-APP: \\ HILFT. WENN DU MITMACHST. \\ Jeizt die Corono-Warn-App herunterladen und Corona gemeinsam bekämplen.}

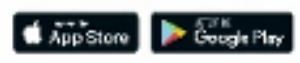

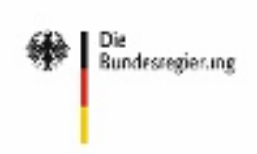

Abb. 2: Bildwortmarke der Bundesregierung ${ }^{22}$

\section{References}

1. Ferretti, Luca et al.: „Quantifying SARS-CoV-2 Transmission Suggests Epidemic Control with Digital Contact Tracing", in: Science 368.6491 (2020). https://doi.org/10.1126/science.abb6936.

2. Quelle: https://styleguide.bundesregierung.de/sgde/basiselemente/programmmarken/corona-warn-app-baukasten/corona-warnappp-der-baukasten-fuer-unterstuetzerinnen-und-unterstuetzer-1756534\#tar-1

3. Während das Mobiltelefon im letztgenannten Fall als Sensor für BluetoothSignale fungiert, operieren andere Lokalisierungstechniken $z$. B. durch GPS oder Funkzellenabfrage.

4. „Bundesregierung wirbt für App zur Corona-Eindämmung“, Hande/sblatt vom 27. März 2020, abrufbar unter:

https://www.handelsblatt.com/politik/deutschland/digitale-virusabwehrbundesregierung-wirbt-fuer-app-zur-corona-eindaemmung/25689956. html (4. Januar 2021).

5. "Internetwirtschaft will mit Kampagne für Corona-Warm-App werben“, Handelsblatt vom 3. April 2020, abrufbar unter:

https://www.handelsblatt.com/technik/medizin/kampf-gegen-pandemieinternetwirtschaft-will-mit-kampagne-fuer-corona-warn-appwerben/25711442.html?ticket=ST-22130392-vJRtEzgynCHJkvivwX6T-ap3 (4. Januar 2021). 
6. „Apps im Kampf gegen COVID-19 und ihre Risiken“, Deutschlandfunk vom 4. April 2020, abrufbar unter: https://www.deutschlandfunk.de/smartphone-tracking= apps-im-kampf-gegen-covid-19-und-ihre.684.de.html?dram:article id=474053 (4. Januar 2021).

7. „Mit Apps gegen die Pandemie“, tagesschau.de vom 22. März 2020, abrufbar unter: https://www.tagesschau.de/inland/coronavirus-forschung-bab-101.html (4. Januar 2021).

8. Containment Scouts sind laut Website des RKI ,in der Regel Studierende der Medizin oder anderer Gesundheitswissenschaften, die [...] vor Ort in den Gesundheitsämtern arbeiten und insbesondere dabei helfen sollen, Kontaktpersonen schneller und effektiver nachzuverfolgen [...] [sowie] auch andere Aufgaben wie beispielsweise die Betreuung von Beratungshotlines zu COVID-19 übernehmen." Abrufbar unter: https://www.rki.de/SharedDocs/FAQ/NCOV2019/FAQ_Scout.html (1. Februar 2021).

9. Bateson, Gregory: „Form, Substance, and Difference”, in: Steps to an Ecology of Mind: Collected Essays in Anthropology, Psychiatry, Evolution, and Epistemology. University of Chicago Press 2000, S. 459. https://doi.org/10.7208/chicago/9780226924601.001.0001. Für ein entsprechendes Verständnis von Verwaltungstechniken der Pandemieüberwachung vgl. Opitz, Sven: „Informing Life: Temporal Politics of Information in the Administration of Pandemics", in: Lyana Francot-Timmermans und Luigi Corrias (Hg.): Out of Joint: Temporal Boundaries of Law and Politics, Routledge 2018, S. 170-191. https://doi.org/10.4324/9781351103480-10.

10. Luhmann, Niklas: „Opportunismus und Programmatik in der öffentlichen Verwaltung“, in: Politische Planung. Aufsätze zur Soziologie und Politik von Verwaltung. VS 1971, S. 165-180, hier S. 165. https://doi.org/10.1007/978-3-66307662-9_10.

11. Luhmann, Niklas: „Lob der Routine“, in: Politische Planung. Aufsätze zur Soziologie und Politik von Verwaltung. VS 1971, S. 113-142, hier S. 122. https://doi.org/10.1007/978-3-663-07662-9_8.

12. Ebd., S. 117.

13. Ebd., S. 129.

14. Vgl. Erikson, L.: „Cell Phones $\neq$ Self and Other. Problems with Big Data Detection and Containment During Epidemics", in: Medical Anthropology Quarterly 32.3 (2018): S. 315-339. https://doi.org/10.1111/maq.12440.

15. Zur Bedeutung unbestimmter Kommunikation vgl. Müller, Julian: Bestimmbare Unbestimmtheiten. Skizze einer indeterministischen Soziologie, Fink 2015, S. $164 \mathrm{ff}$.

16. Latour, Bruno: Eine neue Soziologie für eine neue Gesellschaft. Einführung in die Akteur-Netzwerk-Theorie, Suhrkamp 2007: S. 102. Entsprechend heißt es mit überdeutlichen Anklängen an Bateson: „Ein (...) Handlungsträger, der keinen Unterschied macht, keine Veränderung hervorruft, keine Spur hinterlässt und in keinen Bericht eingeht, ist kein Handlungsträger (...).“ (ebd., S. 92) 
17. Opitz, Sven: „Luftsicherheitszonen: Atmosphären des Selbst in Zeiten von COVID-19", in: Michael Volkmer und Karin Werner (Hg.): Die CoronaGesellschaft. Analysen zur Lage und Perspektiven für die Zukunft, Transcript 2020, S. 125-134, hier S. 126. https://doi.org/10.14361/9783839454329-013.

18. Engemann, Christoph: „Pandemic Media: On the Governmediality of Coronaapps", in: Keidl, Philipp Dominik/Melamed, Laliv (Hg.): Pandemic Media, Meson Press 2020, abrufbar unter: https://pandemicmedia.meson.press/chapters/technologiesmaterialities/pandemic-media-on-the-governmediality-of-corona-apps/ (3. Januar 2021).

19. Zum Nutzungsverhalten vgl. die Studie von Kai T. Horstmann et. al.: „Short Report: Who Does or Does Not Use the 'Corona-Warn-App' and Why?", in: European Journal of Public Health 2020, ckaa239, https://doi.org/10.1093/eurpub/ckaa239

20. Foucault, Michel: Die Sorge um sich. Sexualität und Wahrheit 3, Suhrkamp 1989, S. 92.

21. Ebd., S. 74.

22. Quelle: https://styleguide.bundesregierung.de/sgde/basiselemente/programmmarken/corona-warn-app-baukasten/corona-warnappp-der-baukasten-fuer-unterstuetzerinnen-und-unterstuetzer-1756534\#tar-1

SUGGESTED CITATION: Opitz, Sven; Hall, Kevin; Scheuermann, Klaus: Ein Unterschied, der keinen Unterschied macht? Das Signal der Corona Warn-App und die Verwaltung der Pandemie, in: KWI-BLOG, [https://blog.kulturwissenschaften.de/einunterschied-der-keinen-unterschied-macht/], 08.02.2021

DOI: https://doi.org/10.37189/kwi-blog/20210208-0830 


\section{DuEPublico}

Duisburg-Essen Publications online

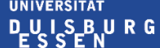

offen im Denken

Dieser Text wird über DuEPublico, dem Dokumenten- und Publikationsserver der Universität Duisburg-Essen, zur Verfügung gestellt. Die hier veröffentlichte Version der EPublikation kann von einer eventuell ebenfalls veröffentlichten Verlagsversion abweichen.

DOI: $\quad 10.37189 / \mathrm{kwi}-\mathrm{blog} / 20210208-0830$

URN: urn:nbn:de:hbz:464-20210208-104021-2

Alle Rechte vorbehalten. 